ARTICLE

DOI: $10.1038 / s 41467-017-01639-5$

\title{
Heterogeneity in the Drosophila gustatory receptor complexes that detect aversive compounds
}

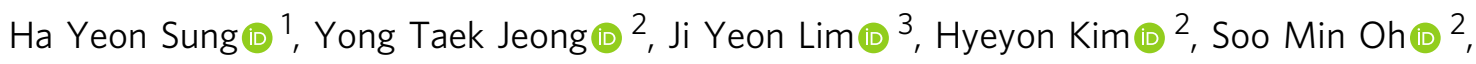
Sun Wook Hwang (ib ${ }^{3}$, Jae Young Kwon (i) ${ }^{1} \&$ Seok Jun Moon (i) ${ }^{2}$

Animals must detect aversive compounds to survive. Bitter taste neurons express heterogeneous combinations of bitter receptors that diversify their response profiles, but this remains poorly understood. Here we describe groups of taste neurons in Drosophila that detect the same bitter compounds using unique combinations of gustatory receptors (GRs). These distinct complexes also confer responsiveness to non-overlapping sets of additional compounds. While either GR32a/GR59c/GR66a or GR22e/GR32a/GR66a heteromultimers are sufficient for lobeline, berberine, and denatonium detection, only GR22e/GR32a/GR66a responds to strychnine. Thus, despite minimal sequence-similarity, Gr22e and Gr59c show considerable but incomplete functional overlap. Since the gain- or loss-of-function of Gr22e or Gr59c alters bitter taste response profiles, we conclude a taste neuron's specific combination of Grs determines its response profile. We suspect the heterogeneity of Gr expression in Drosophila taste neurons diversifies bitter compound detection, improving animal fitness under changing environmental conditions that present a variety of aversive compounds.

\footnotetext{
${ }^{1}$ Department of Biological Sciences, Sungkyunkwan University, Suwon, Gyeonggi-do 16419, Korea. ${ }^{2}$ Department of Oral Biology, BK21 PLUS Project, Yonsei University College of Dentistry, Yonsei-ro 50-1, Seodaemun-gu, Seoul 03722, Korea. ${ }^{3}$ Department of Biomedical Sciences and Department of Physiology, Korea University College of Medicine, Seoul 02841, Korea. Ha Yeon Sung, Yong Taek Jeong and Ji Yeon Lim contributed equally to this work.

Correspondence and requests for materials should be addressed to J.Y.K. (email: jykwon@skku.edu) or to S.J.M. (email: sjmoon@yuhs.ac)
} 
A nimals use their sense of taste mainly for evaluating food quality. Animals detect potentially harmful compounds in their food to avoid ingesting toxic chemicals. Many toxic compounds taste bitter and induce aversive behaviors. Since many plants defend themselves against herbivory by producing a diversity of bitter compounds, many herbivorous insects have evolved gustatory systems capable of detecting large numbers of bitter compounds.

Higher concentrations of these bitter compounds elicit more robust behavioral aversion, suggesting bitter-responsive cells discriminate concentration ${ }^{1}$. It remains unclear, however, whether the bitter taste modality perceives a generic "bitterness" leading to a generic behavioral aversion or whether the system shows more specific molecular discernment permitting more subtle, complex behavioral responses. The bitter-responsive cells of several species reportedly express heterogeneous groups of bitter receptors 2,3 and they respond to distinct bitter compounds ${ }^{3-5}$. Still, the consequences of this heterogeneity of bitter receptor expression have not been fully explored at the molecular and cellular levels.

Drosophila have taste organs all over the body including the labellum, legs, pharynx, anterior wing margin, and even the female genitalia ${ }^{6,7}$. Of these locations, the Drosophila labellum is an attractive place to study bitter taste receptor heterogeneity. The bitter-responsive taste neurons of the labellum are easily accessible for electrophysiological analyses, and we have many genetic reagents that permit precise control over bitter receptor expression in the bitter-responsive taste neurons. Each halflabellum contains 31 taste sensilla classified by their relative length and location into Large (L)-type, Intermediate (I)-type, and Small (S)-type ${ }^{8}$. According to electrophysiological analyses of these 31 taste bristles, all three types of sensilla show distinct response profiles to bitter compounds ${ }^{3}$. After mapping these response profiles to the various morphologic classes, it became clear that the labellar taste sensilla should actually be divided into five classes (Fig. 1a) ${ }^{3}$. L-type and S-c sensilla show almost no response to bitter compounds. S-a and S-b sensilla are broadly tuned, with S-b sensilla showing robust responses to most bitter compounds. I-a and I-b sensilla are narrowly tuned, with complementary response profiles to bitter compounds ${ }^{3}$.

Several classes of chemosensory receptors have been suggested as taste receptors for the detection of aversive chemicals ${ }^{9-11}$, but most bitter compound detection requires members of the gustatory receptor (GR) family. The $60 \mathrm{Gr}$ genes in the fly genome encode 68 proteins by alternative splicing ${ }^{12}, 13$. The bitter gustatory receptor neurons (GRNs) of each sensilla class (i.e., S-a, Sb, I-a, and I-b) express distinct $G r$ subsets $^{3}$. For example, while bitter GRNs in S-a sensilla co-express 29 bitter Grs, bitter GRNs in I-a sensilla express a combination of 6 bitter Grs.

The role the Grs play in taste has been extensively studied by in vivo loss-of-function ${ }^{14-20}$, but those experiments have limitations. Most of those studies focused on flies carrying mutations in individual Grs, measuring the bitter response profiles of very limited numbers of sensilla. Since bitter GRN response profile and $G r$ expression profile are different depending on sensilla type and since multiple independent GRs are required for bitter compound detection ${ }^{20}$, it seems reasonable to assume the function of a given $\mathrm{Gr}$ depends on the Grs with which it is coexpressed. Indeed, in vivo misexpression of an individual $\mathrm{Gr}$ in different bitter GRNs induces differential effects, likely due to the other $G r s$ they express ${ }^{21}$. Still, the precise molecular and cellular role Grs play in the detection of aversive chemicals remains unclear.

In this study, we attempt to parse the function of the bitter Grs in Drosophila in various molecular and cellular contexts. We found that while loss of either Gr32a or Gr66a abolishes sensitivity to lobeline (LOB), berberine (BER), and denatonium (DEN) in all labellar sensilla types, mutation of either Gr22e or Gr59c selectively impairs the detection of these chemicals in the S-b or I-a sensilla, respectively. Misexpression of either GR32a/ GR59c/GR66a or GR22e/GR32a/GR66a confers sensitivity to LOB, BER, and DEN on sweet GRNs and Drosophila S2 cells. We also found Gr22e expression in Gr59c mutant GRNs and Gr59c expression in Gr22e mutant GRNs rescues their detection of LOB, BER, and DEN. This suggests these two Grs are functionally redundant except for the additional detection of strychnine (STR) by the GR22e/GR32a/GR66a complex. Overexpression or misexpression of either Gr22e or Gr59c confers hypersensitivity or novel responsiveness to their respective agonists. The Gr22e, Gr59c double mutant shows a more severe defect in LOB, BER, or DEN avoidance than either the Gr22e or Gr59c single mutant. This may reflect an underlying potential for the graded modulation of repulsion to aversive chemicals. We have found that the detection of the same bitter compounds can be accomplished in different bitter GRNs by distinct bitter GR complexes. Since it is the specific combination of GRs expressed by each bitter GRN that determines its response profile, we propose the heterogeneity of bitter $\mathrm{Gr}$ expression across bitter GRNs diversifies bitter coding and broadens the behavioral repertoire with which insects respond to their chemical environment.

\section{Results}

Minimal receptors for bitter compound detection. Labellar sensilla fall into five distinct functional classes depending on their electrophysiological response profiles (Fig. 1a) ${ }^{3}$. Of these, the I-a sensilla respond specifically to the aversive chemicals LOB, BER, and DEN (Fig. 1b). The bitter GRNs in I-a sensilla express six Grs: Gr32a, Gr33a, Gr39a.a, Gr59c, Gr66a, and Gr89a (Fig. 1b). $\mathrm{We}$, therefore, asked whether these six GRs are sufficient to make functional LOB, BER, and DEN receptors, and if so, what the minimal GR components for LOB, BER, and DEN detection are. Since L-type sensilla do not respond to aversive chemicals ${ }^{8}$, they are a convenient cell type in which to measure the responsiveness to aversive compounds conferred by misexpression of bitter $\mathrm{Gr}$ candidates.

To determine whether the combination of Gr32a, Gr33a, Gr39a.a, Gr59c, Gr66a, and Gr89a is sufficient for LOB, BER, and DEN detection, we misexpressed all six Grs in the sweet GRNs of L-type sensilla using Gr64f-GAL4 (Fig. 1b) ${ }^{22}$ and confirmed the misexpression of each $G r$ in the sweet GRNs by quantitative PCR (Supplementary Table 1). Importantly, control L-type sensilla respond only to sucrose, not to aversive chemicals (Fig. 1c, d). Although misexpression of all six Grs in L-type sensilla has no effect on sucrose responses (Fig. 1d), it confers robust responsiveness to LOB, BER, and DEN. Consistent with the response profile of I-a sensilla, misexpression of all six Grs in L-type sensilla does not confer responsiveness to caffeine (CAF), STR, sucrose octaacetate (SOA), or umbelliferone (UMB) (Fig. 1d). In further validation of these results, we found L-type sweet GRNs co-expressing all six Grs respond to LOB, BER, and DEN in a dose-dependent manner (Supplementary Fig. 1a).

We next sought to identify which Grs are indispensable in the detection of LOB, BER, and DEN. To do so, we misexpressed in the sweet GRNs of L-type sensilla six groups of five Grs at a time, omitting one of the original six Grs per group. We found the absence of Gr32a, Gr59c, or Gr66a prevents ectopic responses to LOB, BER, and DEN, suggesting these three Grs are essential for LOB, BER, and DEN detection (Fig. 1e). We next found misexpression of GR32a, GR59c, and GR66a together confers sensitivity to LOB, BER, and DEN (Fig. 1f), but not CAF, STR, SOA, or UMB. This suggests GR32a/GR59c/GR66a together form 
a

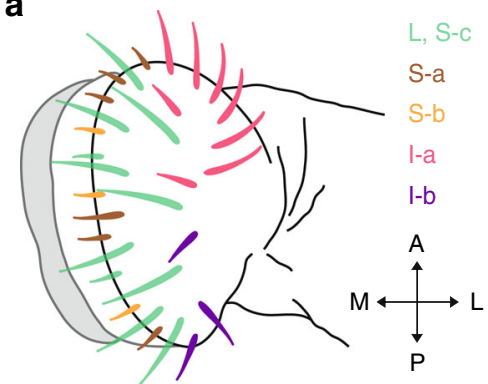

C
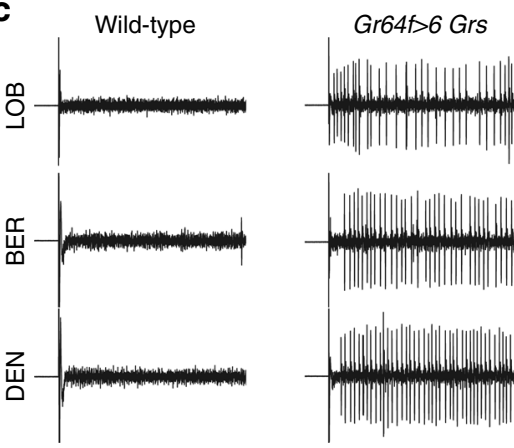

e
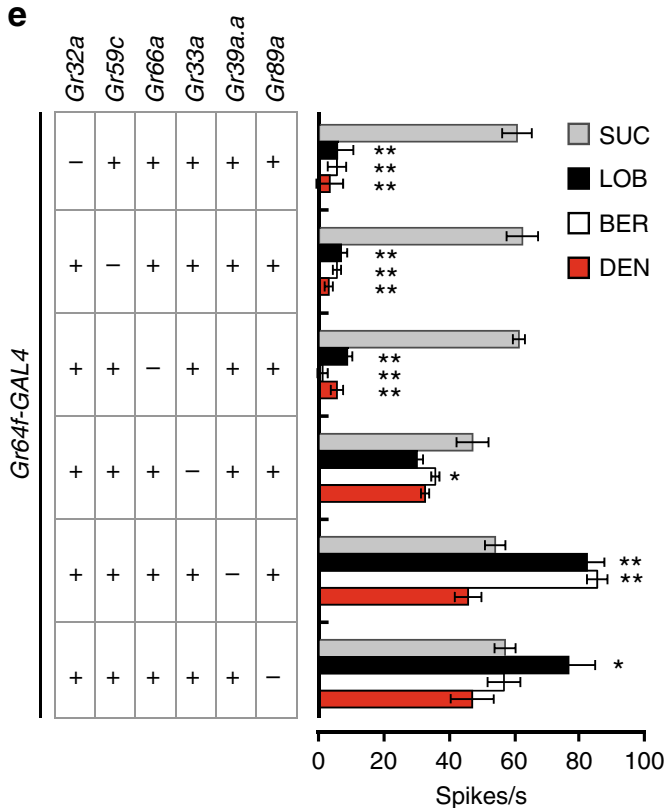

b

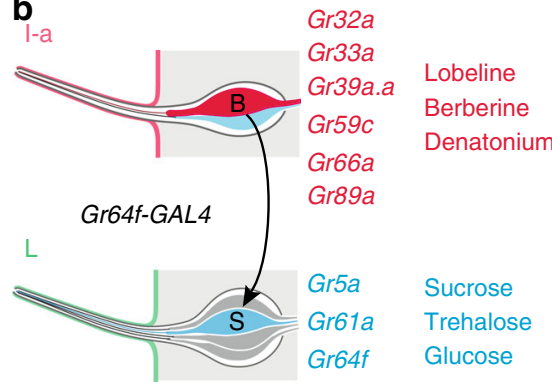

d

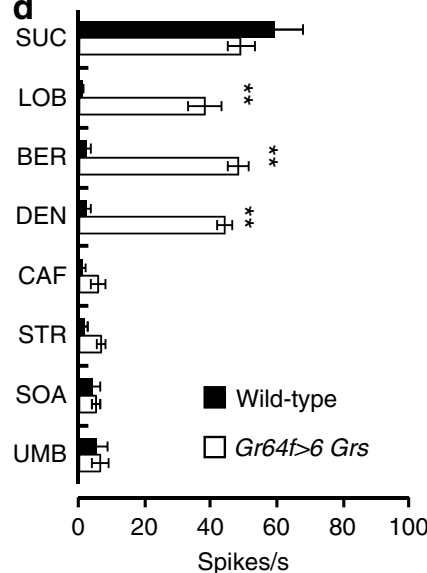

f

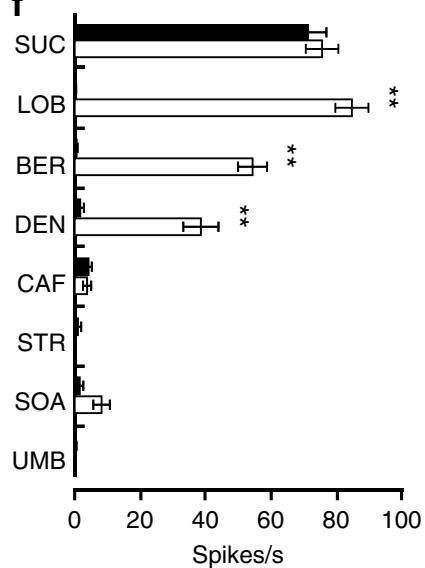

UAS-Gr32a,Gr66a,Gr59c

$\square$ Gr64f>Gr32a,Gr66a,Gr59c

Fig. 1 Identification of Grs for LOB, BER, and DEN detection. a Spatial localization of each class of taste bristle in the labellum. Figure modified from Weiss et al. $^{3}$ (2011). b Schematic for the misexpression of bitter Grs from l-a sensilla in the sweet GRNs of L-type sensilla using Gr64f-GAL4. The Grs normally expressed in bitter GRNs of I-a sensilla and their bitter ligands (red), as well as the sweet Grs normally expressed in sweet GRNs of L-type sensilla and their sugar ligands (blue) are indicated. c Representative traces from L-type sensilla of flies of the indicated genotypes upon application of $1 \mathrm{mM}$ stimuli of LOB, BER, or DEN. d Mean responses of L-type sensilla induced by the indicated chemicals: $100 \mathrm{mM}$ sucrose (SUC), $1 \mathrm{mM}$ LOB, $1 \mathrm{mM}$ BER, $1 \mathrm{mM}$ DEN, $5 \mathrm{mM}$ CAF, $1 \mathrm{mM} \mathrm{STR}, 1 \mathrm{mM}$ SOA, and $1 \mathrm{mM} \mathrm{UMB} . n=6-10$. Unpaired $t$-test or the Mann-Whitney $U$-tests as appropriate. e Identification of the Grs required for LOB, BER, and DEN detection. The mean responses of L-type sensilla of the indicated genotypes upon application of 1 mM stimuli of LOB, BER, and DEN as well as $100 \mathrm{mM}$ SUC are shown. $n=6-10$. ANOVAs followed by Dunnet T3 post hoc tests for LOB and the Kruskal-Wallis tests followed by Mann - Whitney $U$ post hoc tests for BER and DEN. The asterisks indicate statistically significant differences from Gr64f $>6$ Grs flies in $\mathbf{d}$. $\mathbf{f}$ The mean responses of L-type sensilla expressing Gr32a, Gr59c, and Gr66a induced by the indicated chemicals. $n=6-10$. Unpaired Student's $t$-tests or the Mann-Whitney U-tests as appropriate. All data are presented as means \pm S.E.M. ${ }^{\star} p<0.05,{ }^{\star \star} p<0.01$. Complete genotypes are listed in Supplementary Table 6

a receptor for LOB, BER, and DEN. Since neither the expression of any one of these three Grs nor any combination of two of these three Grs (i.e., Gr32a/Gr66a, Gr32a/Gr59c, or Gr66a/Gr59c) is sufficient to confer LOB, BER, and DEN sensitivity, GR32a, GR59c, and GR66a are the minimal and essential components for
LOB, BER, and DEN detection (Supplementary Fig. 1b). It is noteworthy that misexpression of Gr32a, Gr59c, and Gr66a did not affect the sucrose responses of sweet GRNs, even as it conferred LOB, BER, and DEN responses (Fig. 1d-f). Furthermore, misexpression of Gr32a, Gr59c, and Gr66a in the sweet 
a

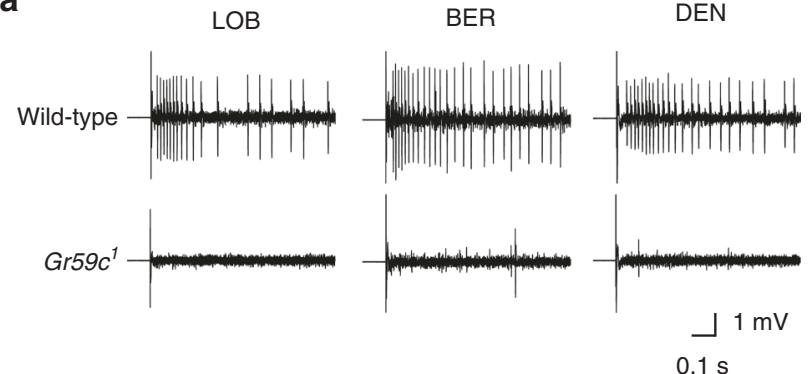

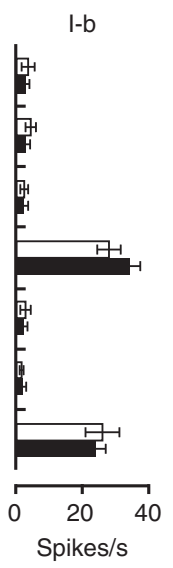

$\square$ Wild-type

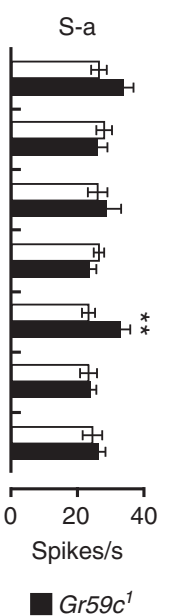

S-b

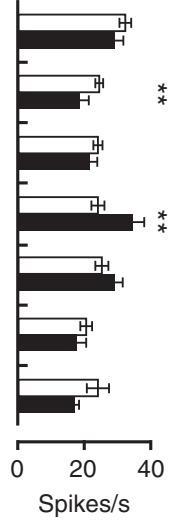

Fig. 2 Electrophysiological responses of Gr59 $c^{7}$ mutant sensilla to bitter chemicals. a Representative traces from 15 sensilla from wild-type and Gr59c flies upon application of $1 \mathrm{mM}$ stimulations with the indicated chemicals. $\mathbf{b}$ Mean responses of the indicated sensilla in wild-type and Gr59c ${ }^{7}$ flies to $1 \mathrm{mM}$ stimuli of the indicated bitter compounds except $5 \mathrm{mM}$ CAF. $n=6-50$. Unpaired Student $t$-tests or Mann-Whitney U-tests as appropriate. All data are presented as means \pm S.E.M. ${ }^{\star \star} p<0.01$

GRNs of a new Gr64 cluster deletion mutant (Gr64af) covering the coding regions of the entire Gr64 cluster conferred a similar level of sensitivity to LOB, BER, and DEN as that in wild-type sweet GRNs (Supplementary Figs. 1c, 2a). Previous mutants of the Gr64 cluster either deleted additional neighboring genes or only deleted some genes of the cluster ${ }^{22-25}$, and were thus less suitable for this purpose. Loss of six of the nine sweet clade Grs does not affect ectopic responses to LOB, BER, and DEN upon Gr32a, Gr59c, and Gr66a expression in sweet GRNs. This indicates that the ectopic responses to LOB, BER, and DEN we observed are not due to erratic interactions between endogenous sweet clade GRs and misexpressed bitter GRs.

I-a sensilla require $G r 59 c$ for bitter compound response. None of the labellar sensilla of Gr32a and Gr66a mutant flies show a significant electrophysiological response to LOB, BER, or DEN (Supplementary Fig. 1d) ${ }^{15}$. We asked whether loss of $G r 59 c$ also impairs sensitivity to LOB, BER, and DEN by generating a Gr59c mutant $\left(G r 59 c^{1}\right)$ by ends-out homologous recombination (Supplementary Fig. 2f) ${ }^{26}$. As expected, the I-a sensilla of Gr59c mutant flies do not respond to LOB, BER, or DEN (Fig. 2a, b). The S-a and S-b sensilla of $G r 59 c^{1}$ flies, which do not express $G r 59 c$, show wild-type responses to LOB, BER, and DEN. This suggests other receptor complexes are responsible for LOB, BER, and DEN sensitivity in these sensilla (Fig. 2b). In addition, we found $G r 59 c^{1}$ flies show enhanced responses to CAF, STR, and UMB (Fig. 2b). This is consistent with a previous report that the I-a sensilla of $G r 59 c$ mutant flies show increased sensitivity to

$\mathrm{CAF}$ and $\mathrm{UMB}^{21}$. In addition to confirming this, we found loss of Gr59c also increases the sensitivity of S-a sensilla to STR and S-b sensilla to CAF.

Identifying Grs for bitter compound sensing in S-b sensilla. S-b sensilla detect LOB, BER, and DEN in the absence of $G r 59 c$, suggesting another GR complex recognizes these compounds in these sensilla. We, therefore, set out to identify the bitter Grs required for LOB, BER, and DEN detection in S-b sensilla. Bitter GRNs in S-b sensilla express 16 different $G r s^{3}$. While Gr32a, Gr33a, and Gr66a are required for the detection of LOB, BER, and $\mathrm{DEN}^{15,19}, \mathrm{Gr} 8 a$ is not ${ }^{14}$. To investigate the rest of these 16 $G r s$, we obtained three $G r$ mutants that cover five Grs (i.e., Gr22e, Gr28b.a, Gr28b.d, Gr28b.e, Gr36c) ${ }^{27}$ and then generated five more mutants (i.e., Gr22f $f^{1}, G r 28 a^{1}, G r 36 a^{1}, G r 39 b^{1}, G r 89 a^{1}$ ) by endsout homologous recombination (Supplementary Fig. 2$)^{26}$. We then examined the responses of their S-b sensilla to LOB, BER, and DEN. We were forced to exclude Gr39a.a and Gr59a; the Gr39a.a mutant is adult-lethal, and our attempts at obtaining a Gr59a mutant were unsuccessful.

Consistent with previous reports ${ }^{15,19}$, loss of Gr32a, Gr33a, or Gr66a nearly abolishes LOB, BER, and DEN-evoked action potentials in S-b sensilla (Fig. $3 a-c$ ). In addition, we found the S-b sensilla of Gr22e mutant flies show significantly reduced responses to LOB, BER, and DEN (Fig. 3a-d), whereas those of $G r 22 f$ and $G r 39 b$ mutants show selectively reduced responses to DEN (Fig. 3c).

S-b sensilla required Gr22e for bitter compound response. Next, we sought a more detailed picture of the role Gr22e plays in the detection of aversive compounds. In addition to their reduced sensitivity to LOB, BER, and DEN, Gr22e mutant S-b sensilla show lower sensitivity to STR and SOA (Fig. 3e). Gr22e mutant $\mathrm{S}$-a sensilla are less sensitive to DEN and STR (Fig. 3e).

Since S-b sensilla lacking Gr22e show reduced responses to LOB, BER, DEN, STR, and SOA, we asked whether GR22e, together with GR32a and GR66a, forms the functional receptor for these bitter compounds. Misexpression of Gr22e, Gr32a, and Gr66a using Gr64f-GAL4 does not alter sucrose responses but it does confer sensitivity to LOB, BER, DEN, and STR on the sweet GRNs of L-type sensilla (Fig. 3f). We also found ectopic coexpression of Gr22e, Gr32a, and Gr66a in sweet GRNs lacking the Gr64 cluster (Gr64af) confers sensitivity to LOB, BER, DEN, and STR, indicating GR22e/GR32a/GR66a represents the minimal complex necessary for LOB, BER, DEN, and STR responses (Supplementary Fig. 3a). Since loss of Gr22f and Gr39b reduces the responses of S-b sensilla to DEN, we also asked whether Gr22f and Gr39b participate in the DEN receptor. Similar misexpression of either Gr22f or Gr39b with both Gr32a and Gr66a does not confer DEN sensitivity on the sweet GRNs of L-type sensilla (Supplementary Fig. 3b). This suggests the reduced responses to DEN in the Gr22f and Gr39b mutants are non-specific. They may instead be due to the formation of a DEN-detecting complex with other GRs or to an indirect reduction in DEN responses.

Gr22e and Gr59c are redundant in bitter compound detection. Since $G r 59 c$ and $G r 22 e$ are expressed in different sensilla and independently participate in receptor complexes that each respond to $\mathrm{LOB}, \mathrm{BER}$, and $\mathrm{DEN}$, we wondered whether Gr22e can substitute for $G r 59 c$ in I-a sensilla and vice versa in S-b sensilla. We, therefore, expressed wild-type Gr22e or $G r 59 c$ in bitter GRNs of Gr22e mutants using Gr33a-GAL4. As expected, Gr22e expression rescues the Gr22e mutant phenotype in S-b sensilla (Fig. $4 \mathrm{a}-\mathrm{d}$ ). In addition, Gr22e and Gr59c expression both induce hypersensitivity to LOB, BER, and DEN in S-a and S-b 

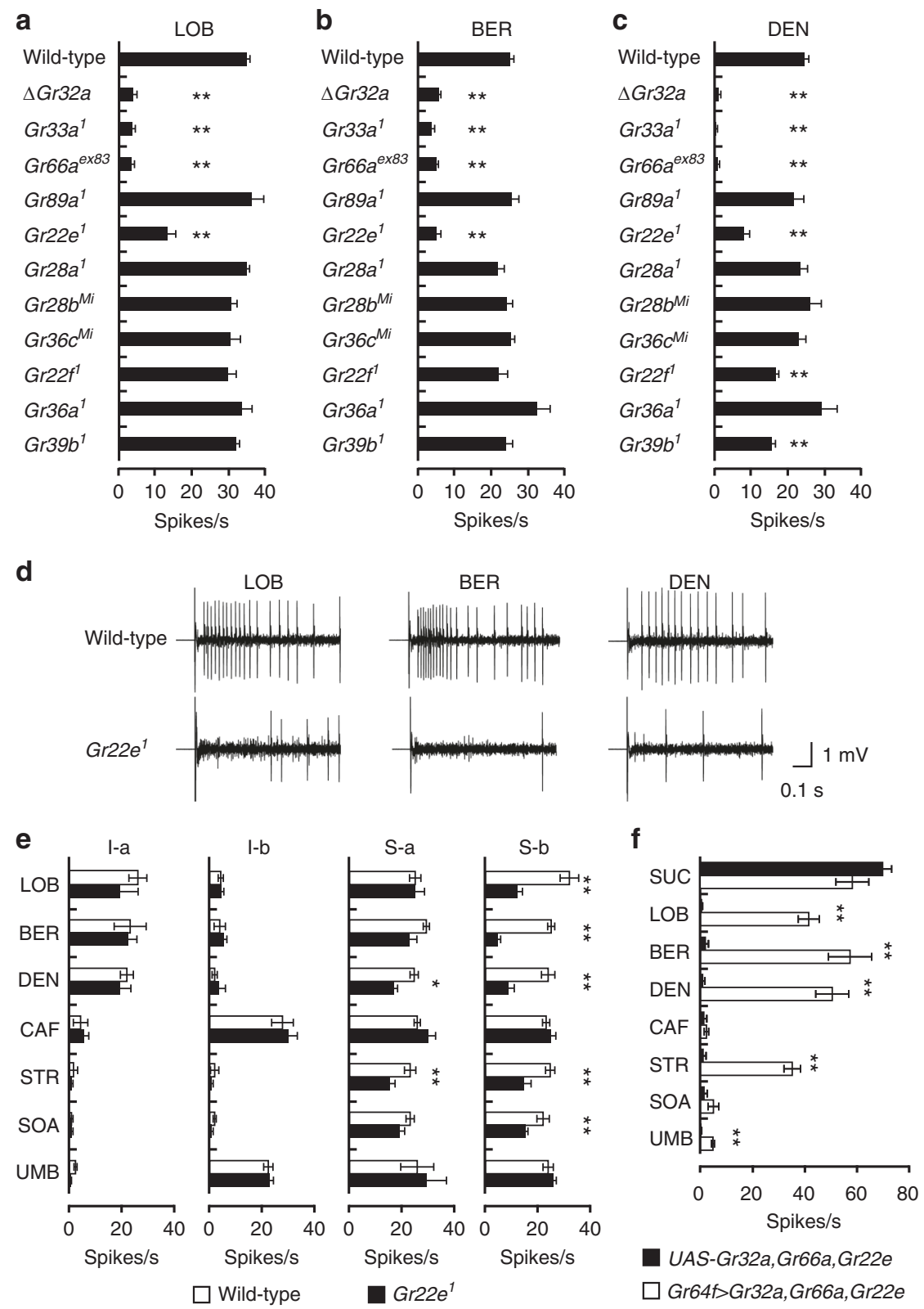

Fig. 3 Identification of Grs for LOB, BER, and DEN detection in S-b sensilla. a-c Screening to identify the Grs required for the responses in S-b sensilla to LOB a, BER $\mathbf{b}$, and DEN c. $1 \mathrm{mM}$ of each chemical was used. $n=8-31$. ANOVAs with Tukey or Dunnett T3 post hoc tests or Kruskal-Wallis tests with Mann-Whitney $U$ post hoc tests as appropriate. The asterisks indicate statistically significant differences from wild-type flies. $\mathbf{d}$ Representative traces from wild-type and Gr22e $e^{7} 5$ sensilla upon application of LOB, BER, and DEN. e Mean responses of the indicated sensilla from wild-type and Gr22 $e^{1}$ flies to $1 \mathrm{mM}$ stimulations with the indicated bitter substances except $5 \mathrm{mM} \mathrm{CAF.} n=6-30$. Unpaired Student's $t$-tests or Mann-Whitney U-tests as appropriate. $\mathbf{f}$ Mean responses of Gr32a, Gr66a, and Gr22e-expressing L-type sensilla induced by $1 \mathrm{mM}$ stimulations with the indicated chemicals except $100 \mathrm{mM}$ SUC and 5 mM CAF. $n=7-14$. Unpaired Student's $t$-tests or Mann-Whitney $U$-tests as appropriate. All data are presented as means \pm S.E.M. ${ }^{\star} p<0.05$, ${ }^{\star \star} p<0.01$. Complete genotypes are listed in Supplementary Table 6

sensilla (Fig. 4a-c). Only Gr22e, however, rescues the STR detection defect of Gr22e $e^{1}$ S-b sensilla and induces STR hypersensitivity in S-a sensilla (Fig. 4d). This suggests the GR22e/ GR32a/GR66a complex detects STR, but the GR59c/GR32a/ GR66a complex does not. Neither misexpression of Gr22e nor overexpression of $G r 59 c$ affects sensitivity to LOB, BER, and DEN in I-a sensilla (Fig. $4 \mathrm{a}-\mathrm{c}$ ), but misexpression of $\mathrm{Gr} 22 e$ results in a novel response to STR in I-a sensilla (Fig. 4d). Misexpression of Gr22e or Gr59c in I-b sensilla results in a novel response to LOB, $\mathrm{BER}$, and DEN and misexpression of Gr22e also results in a novel response to STR in I-b sensilla (Fig. $4 \mathrm{a}-\mathrm{d}$ ).
Next, we expressed either wild-type Gr22e or Gr59c in bitter GRNs of Gr59c mutants using Gr33a-GAL4. Misexpression of Gr22e or Gr59c in the S-a and S-b sensilla of Gr59c mutant flies leads to their hypersensitivity to LOB, BER, and DEN (Fig. 4e-g). Expression of either Gr22e or Gr59c can rescue the defect in LOB, BER, and DEN sensitivity observed in the I-a sensilla of Gr59c mutants (Fig. $4 \mathrm{e}-\mathrm{g}$ ). Misexpression of Gr22e or Gr59c in I-b sensilla results in a novel response to LOB, BER, and DEN (Fig. $4 \mathrm{e}-\mathrm{g}$ ). Only Gr22e overexpression induces STR hypersensitivity in S-a and S-b sensilla and only Gr22e misexpression induces novel responses to STR in I-a and I-b sensilla (Fig. 4h). 

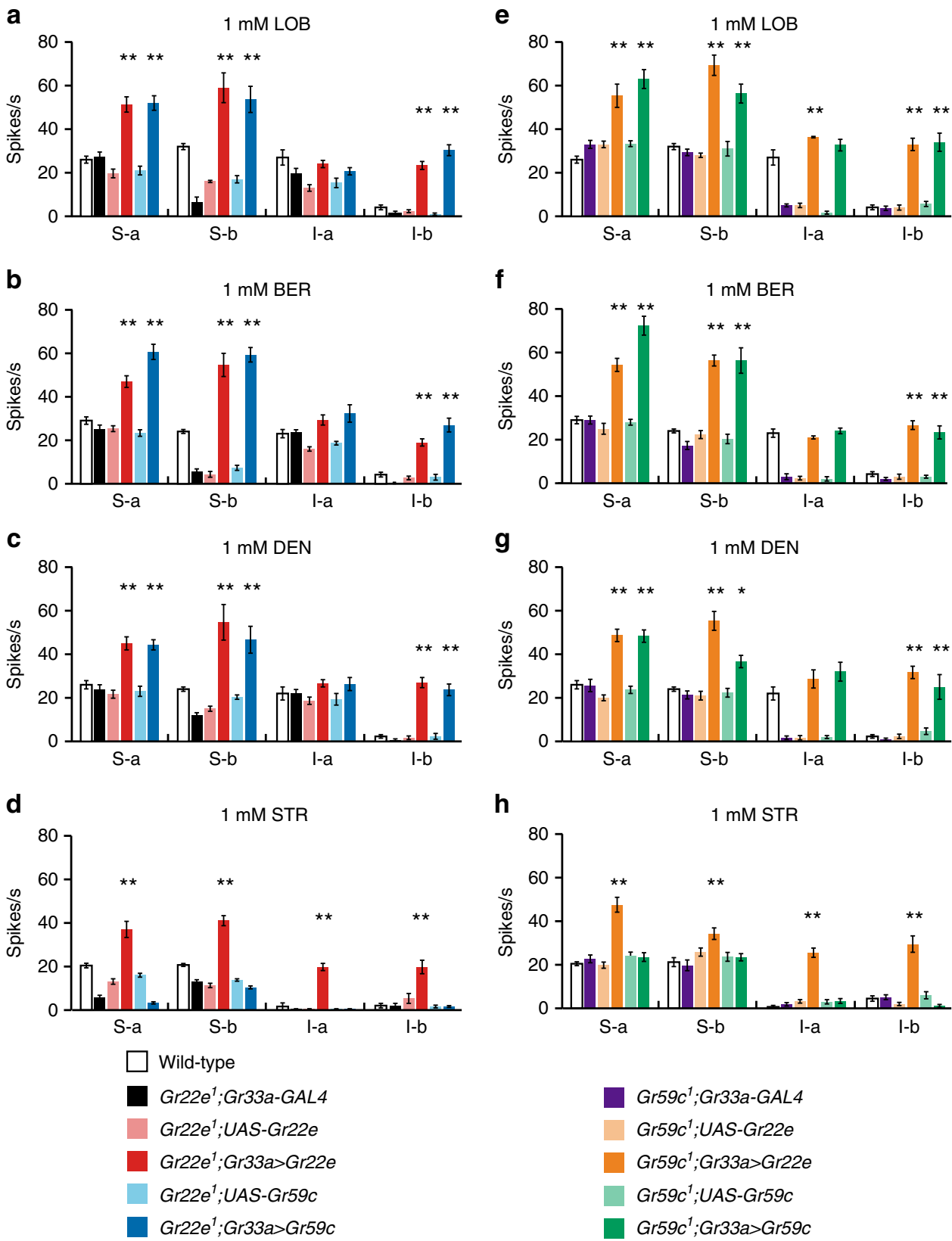

Fig. 4 Functional redundancy of Gr22e and Gr59c. Mean responses in flies of the indicated genotypes evoked by $1 \mathrm{mM}$ stimulations with LOB a, e, BER $\mathbf{b}, \mathbf{f}$, DEN c, $\mathbf{g}$, or STR d, h. $n=6-69$. ANOVAs followed by Tukey or Dunnett T3 post hoc tests or Kruskal-Wallis tests followed by Mann-Whitney U post hoc tests as appropriate. All data are presented as means \pm S.E.M. The asterisks indicate statistically significant differences $\left({ }^{\star} p<0.05,{ }^{\star \star} p<0.01\right)$ from wildtype flies. Complete genotypes are listed in Supplementary Table 6

In summary, expression of Gr22e in the Gr22e mutant and expression of $G r 59 c$ in the Gr59c mutant both rescue their respective mutant phenotypes, confirming the respective defects are attributable to loss of $\mathrm{Gr} 22 e$ or $\mathrm{Gr} 59 \mathrm{c}$. In addition, expression of Gr22e in the Gr59c mutant and Gr59c in the Gr22e mutant produce nearly identical responses to LOB, BER, and DEN. This indicates $G r 22 e$ can substitute for $G r 59 c$ and vice versa, except in the detection of STR.

Conferring bitter chemical-induced currents on S2 cells. To determine whether Gr22e and Gr59c are ion conducting subunits that confer bitter compound sensitivity, we co-expressed Gr22e, Gr32a, and Gr66a or Gr32a, Gr59c, and Gr66a in Drosophila S2 cells and performed whole-cell voltage-clamp recordings. Consistent with our in vivo gain-of-function results, we found both
Gr combinations confer sensitivity to LOB, BER, and DEN on S2 cells (Supplementary Fig. 4). These novel responses, which appear as outwardly rectifying currents in Supplementary Fig. 4, also seem to be dose-dependent. GR22e/GR32a/GR66a-expressing S2 cells also respond to STR, but neither GR22e/GR32a/GR66aexpressing S2 cells nor GR59c/GR32a/GR66a-expressing S2 cells respond to CAF or SOA (Supplementary Fig. $4 \mathrm{e}, \mathrm{j}$ ). These results are consistent with our in vivo loss-of-function and misexpression results.

Bitter compound detection in Gr22e Gr59c double mutants. The S-a sensilla of both Gr22e and Gr59c single mutants show normal responses to LOB, BER, and DEN (Figs. 2, 3). We initially assumed this was a result of the functional redundancy of Gr22e and $G r 59 c$ in the detection of LOB, BER, and DEN in the S-a 

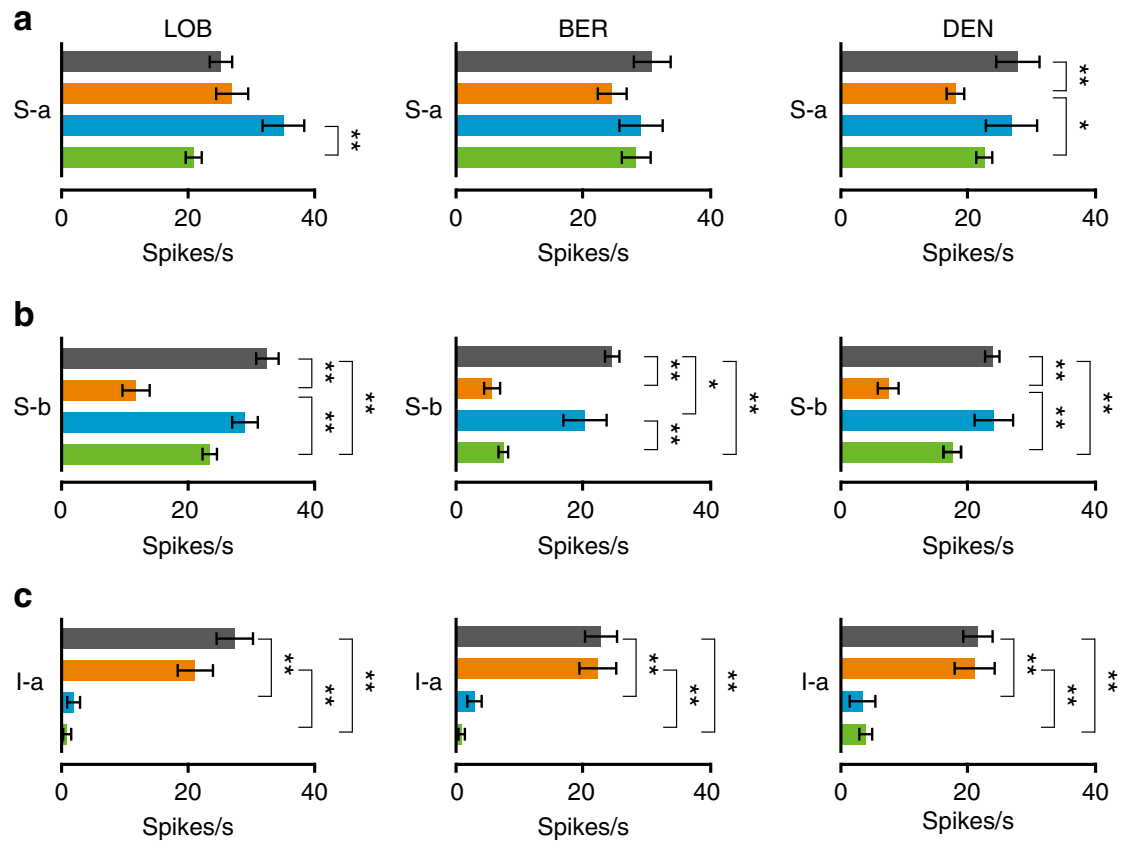

Wild-type

Gr22e $e^{1}$

Gr59c ${ }^{1}$

$G r 22 e^{1}, G r 59 c^{1}$

Fig. 5 Effect of Gr22e and Gr59c mutation on S-a sensilla. Mean response of S-a sensilla of the indicated genotype to $1 \mathrm{mM}$ stimulations with LOB a, BER $\mathbf{b}$, and DEN c. $n=6-54$. ANOVAs followed by Tukey or Dunnett T3 post hoc tests or Kruskal-Wallis tests followed by Mann-Whitney $U$ post hoc tests as appropriate. All data are presented as means \pm S.E.M. The asterisks indicate statistically significant differences $\left({ }^{\star} p<0.05,{ }^{\star \star} p<0.01\right)$

sensilla. To test this possibility, we generated the Gr22e,Gr59c double mutant. When we measured the responses of the S-a sensilla to LOB, BER, and DEN, we found the S-a sensilla in the Gr22e,Gr59c double mutant does not show a significant reduction in response to LOB, BER, and DEN compared to the wild-type or single mutant with the exception of the comparison between the $G r 22 e, G r 59 c$ double mutant and the Gr59c mutant in response to LOB (Fig. 5a). We were particularly surprised to note that while Gr22e mutant S-a sensilla show reduced responses to DEN, those of the Gr22e, Gr59c double mutant do not (Fig. 5a). These results suggest other Grs must play a role in the detection of LOB, BER, and DEN in S-a sensilla.

Next, we measured the LOB, BER, and DEN sensitivity of the $\mathrm{S}$-b and I-a sensilla of the Gr22e,Gr59c double mutants. We found Gr22e,Gr59c double mutant S-b sensilla show reduced sensitivity to LOB, BER, and DEN like Gr22e single mutant S-b sensilla, but their defect in LOB and DEN detection is not as severe (Fig. 5b). $G r 22 e, G r 59 c$ double mutant I-a sensilla, like those of the Gr59c single mutant, do not respond to LOB, BER, or DEN (Fig. $5 \mathrm{c}$ ).

Differential contribution of labellar sensilla to avoidance. Finally, we used a two-way choice assay to ask how the different types of labellar bitter-responsive sensilla contribute to feeding decisions. We gave flies a choice between $1 \mathrm{mM}$ sucrose and 5 $\mathrm{mM}$ sucrose combined with different concentrations of aversive compounds (LOB, BER, and DEN). While wild-type flies demonstrate dose-dependent repulsion to all the bitter compounds we tested (Fig. 6), both Gr22e and Gr59c single mutant flies show reduced repulsion to LOB- or DEN-containing foods (Fig. 6a, c). This defect is even more severe in the Gr22e,Gr59c double mutant (Fig. 6a, c). These data indicate the respective contributions of the S-b and I-a sensilla to behavioral LOB and DEN aversion are independent and additive. Surprisingly, we found that while the Gr59c mutant demonstrates a significant defect in BER avoidance, the Gr22e mutant does not (Fig. 6b).
The Gr22e,Gr59c double mutant, however, shows an even more severe defect in BER avoidance than the Gr59c single mutant (Fig. 6b). These data further suggest the gustatory system uses different types of sensilla expressing different receptors to direct subtly different behavioral responses depending on the bitter compound in question.

\section{Discussion}

Here we have clarified several principles that inform our understanding of the Drosophila Grs that detect aversive compounds. First, three Grs seem to cooperate to form functional bitter compound receptors. We have shown in sweet GRNs and Drosophila S2 cells that co-expression of Gr22e, Gr32a, and Gr66a is sufficient to confer sensitivity to LOB, BER, DEN, and STR, while co-expression of $G r 32 a, G r 59 c$, and Gr66a confers sensitivity to LOB, BER, and DEN. This is reminiscent of our identification of the heterotrimeric L-canavanine receptor-GR8a, GR66a, and GR98 $b^{20}$. If we arrange these bitter GR complex components in decreasing order of tuning breadth from broad to narrow, the resulting order would be: Gr66a, Gr32a, Gr22e, and Gr59c. Second, although Drosophila GR complexes function as heteromultimers, they seem to remain promiscuous. A given GR complex can be activated by multiple bitter chemicals: GR22e/ GR32a/GR66a by LOB, BER, DEN, and STR; GR32a/GR59c/ GR66a by LOB, BER, and DEN. Conversely, the same bitter chemicals can activate several different $\mathrm{Gr}$ complexes. For example, BER activates GR22e/GR32a/GR66a, GR32a/GR59c/ GR66a, and another unknown GR complex in S-a sensilla. Finally, different GRNs use distinct GR combinations even for detecting the same chemicals. S-b sensilla require GR22e/GR32a/ GR66a to detect LOB, BER, and DEN, while I-a sensilla require GR32a/GR59c/GR66a to accomplish the same thing. Since the S-a sensilla of $G r 22 e, G r 59 c$ double mutants remain sensitive to LOB, $\mathrm{BER}$, and DEN, they must have at least one additional receptor complex for these aversive compounds. It is likely S-b sensilla also 

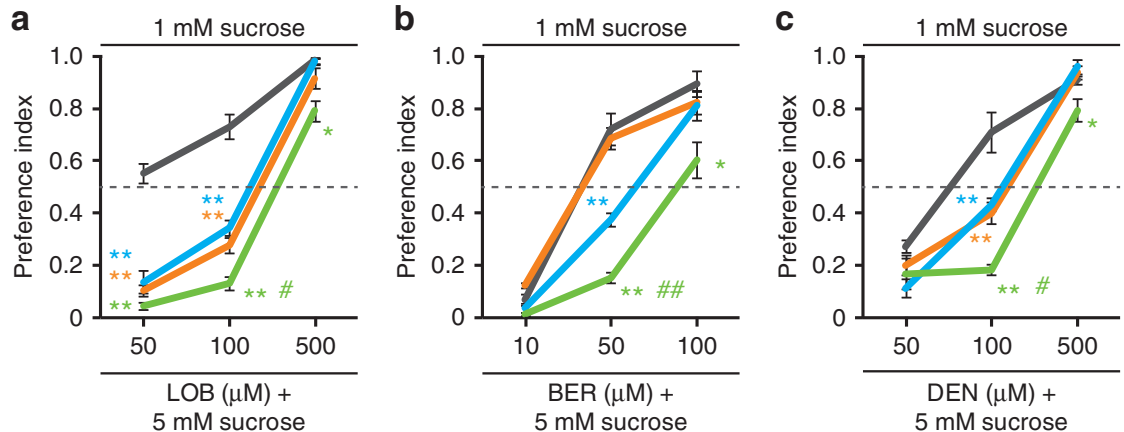

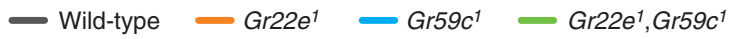

Fig. 6 The contribution of Gr22e and Gr59c to bitter food aversion. Two-way choice assays using the indicated concentrations of a LOB, b BER, and $\mathbf{c}$ DEN. $n$ $=4-12$. ANOVAs followed by Tukey post hoc tests or Kruskal-Wallis tests followed by Mann-Whitney U post hoc tests as appropriate. All data are presented as means \pm S.E.M. The asterisks and sharps indicate statistically significant differences from wild-type flies $\left({ }^{\star} p<0.05\right.$, $\left.{ }^{\star \star} p<0.01\right)$ and from both Gr22e $e^{1}$ and $\operatorname{Gr}_{59} c^{1}\left({ }^{\#} p<0.05,{ }^{\# \#} p<0.01\right)$, respectively

have another receptor complex that responds to $\mathrm{LOB}, \mathrm{BER}$, and DEN, because S-b sensilla in the Gr22e mutant still respond weakly to these chemicals and the S-b sensilla of Gr22e,Gr59c double mutants are more sensitive to these chemicals than those of the Gr22e single mutant, presumably due to increase of expression of unknown LOB, BER, and DEN receptor complexes in the Gr22e,Gr59c double mutants (Fig. 5b).

The fact that Gr22e,Gr59c double mutant S-a sensilla show a near wild-type response to LOB, BER, and DEN (Fig. 5) makes it clear that distinct bitter GR complexes (even in the same GRN) can detect overlapping sets of bitter compounds. What advantage does such a strategy provide? We speculate that this permits specific sensilla to fine tune their responses to bitter compounds. For example, increasing the expression of Gr22e in S-a or S-b sensilla would make them more sensitive to LOB, BER, DEN, and STR. As expected with such a scheme, loss of either Gr22e or Gr59c results in a partial impairment of LOB, BER, and DEN avoidance rather than a complete loss of aversion (Fig. 6). Although we could not exclude the possibility that the behavioral consequences of the loss of Gr22e or Gr59c were influenced by taste organs other than the labellum (e.g., the tarsal taste sensilla) (Fig. 6), this is unlikely because the Gr22e and Gr59c reporters are not expressed in the tarsal taste sensilla ${ }^{28}$. This raises the interesting possibility that distinct GR complexes sense bitter chemicals not only depending on the taste sensilla in which they are expressed but also the different taste organs.

A growing body of evidence suggests interactions between Grs co-expressed in bitter GRNs are an important determiner of the GRN's response profile ${ }^{3,21}$. We found overexpression or misexpression of Gr22e or Gr59c in S-a and S-b sensilla increases the endogenous response to LOB, BER, and DEN (Fig. 4). It is likely the extra GR22e or GR59c proteins bind GR32a and GR66a. This would form more LOB, BER, and DEN receptor complexes, increasing the sensitivity of the response to these chemicals. In contrast, neither misexpression of Gr22e nor overexpression of Gr59c affects LOB, BER, and DEN responses in I-a sensilla. Misexpression of Gr22e or overexpression of Gr59c in the Gr59c mutant results in a complete wild-type level rescue. It is possible that in wild-type I-a sensilla, most GR32a and GR66a molecules are already bound to GR59c, and therefore extra GR22e or GR59c do not form additional LOB, BER, and DEN receptors with GR32a and GR66a. Since misexpression of Gr22e or Gr59c in I-b sensilla confers on them novel sensitivity to LOB, BER, and DEN, it is possible the exogenous GR22e or GR59c proteins are outcompeting other GRs for binding to the endogenous GR32a and GR66a molecules, forming novel LOB, BER, and DEN receptor complexes. If this hypothesis is true, we should see a concurrent reduction in the responses of these I-b sensilla to their normal ligands. Indeed, overexpression of Gr59c suppresses the normal I$\mathrm{b}$ response to CAF, presumably secondary to a reduction in the number of functional CAF receptor complexes ${ }^{3,21}$. In addition, loss of $G r 59 c$ in I-a sensilla, which shifts their bitter response profile to one resembling that of I-b sensilla (Fig. $2 b)^{21}$, allows other GRs expressed in I-a sensilla to form new functional receptor complexes. This then alters their sensitivity to CAF, UMB, TPH, and other bitter compounds (Fig. 2b) ${ }^{21}$.

In summary, our results suggest bitter coding is much more complex and dynamic than expected. In the future, we hope to correlate changes in $\mathrm{Gr}$ expression with changes in environmental conditions and internal physiological states to better understand the dynamics of this system and how they affect animal behavior.

\section{Methods}

Fly stocks. All fly stocks were maintained on standard cornmeal-molasses agar medium at $25^{\circ} \mathrm{C}$ and $60 \%$ humidity under a $12 \mathrm{~h} / 12 \mathrm{~h}$ light/dark cycle, respectively. 70FLP,70I-SceI/CyO (BL6934), Gr28b ${ }^{m i}$ (BL24190), and Gr36c ${ }^{m i}$ (BL26596) were obtained from the Bloomington Drosophila Stock Center. Gr22e ${ }^{1}$ (\#140936) was obtained from the Kyoto Stock Center. To minimize genetic background artifacts, all mutant strains were outcrossed to $w^{1118}$ for five generations. $\Delta G r 32 a$ was a gift from Hubert Amrein. Gr64f-GAL4 and UAS-Gr59c were gifts from John Carlson. $G r 33 a^{1}, G r 66 a^{e x 83}, U A S-G r 33 a, U A S-G r 66 a$, and Gr33a-GAL4 were previously described $^{18,19}$. Gr64af harboring the deletion of the whole Gr64 cluster will be described in a separate manuscript in detail (Supplementary Fig. 2a).

Generation of transgenic flies. UAS-Gr22e, UAS-Gr22f, UAS-Gr32a, UAS-Gr39a $a, U A S-G r 39 b$, and UAS-Gr89a were generated. Labellar cDNAs were synthesized using Thermo Scientific RevertAid Reverse Transcriptase (Thermo Fisher Scientific, Waltham, MA) followed by transcript extraction using TRIZOL (Thermo Fisher Scientific). Individual Gr cDNAs were amplified and cloned from total labellar cDNAs using primer pairs listed in Supplementary Table 2. The amplified DNA fragments were inserted into pUAST vectors via conventional molecular cloning methods. pUAST vectors carrying each $G r$ cDNA were injected into $w^{1118}$ embryos by standard transgenesis techniques (BestGene Inc., Chino Hills, CA).

Generation of Gr mutants. Gr22f $f^{1}, G r 28 a^{1}, G r 36 a^{1}, G r 39 b^{1}, G r 59 c^{1}$, and $G r 89 a^{1}$ were generated by homologous recombination ${ }^{26}$. In general, $3 \mathrm{~kb} 5^{\prime}$ and $3^{\prime}$ homology arms for each Gr coding region were amplified by genomic DNA PCR using primer pairs listed in Supplementary Table 3 and cloned into mutant construction vectors. pw35 was used for Gr22f $f^{1}, G r 36 a^{1}, G r 39 b^{1}$, and $G r 89 a^{1}$. pw35GAL4 and pw35+ were used for $G r 28 a^{1}$ and $G r 59 c^{1}$, respectively. After obtaining transformants carrying each targeting vector, the transgenes were mobilized by crossing to $70 F L P, 70 I-S c e I / C y O$. Mosaic-eyed progeny (F1) were crossed to $w^{1118}$ to obtain red-eyed F2 progenies, which were subjected to PCR analysis $^{29}$. Each $\mathrm{Gr}$ mutation was confirmed by genomic PCR using primers listed in Supplemental Table 4. 
Quantitative PCR. Total RNA was extracted from dissected labella using TRIZOL (Thermo Fisher Scientific) according to the manufacturer's instructions. Labellar cDNA was synthesized from $1 \mu \mathrm{g}$ of total RNA with the RevertAid reverse transcriptase system (Thermo Fisher Scientific). Quantitative PCR was conducted with a Quantstudio3 real-time PCR instrument (Thermo Fisher Scientific) using the ABI SYBR green system (Applied Biosystems, Foster City, CA). $\mathrm{C}_{\mathrm{T}}$ values were measured in triplicate. The sequence of the primer pairs for each $G r$ are shown in Supplementary Table 5. The concentrations of the linearized DNA carrying each of 6 Grs were measured with a Nanodrop (Thermo Fisher Scientific) and a series of 10 -fold dilutions of linearized DNA were subjected to quantitative PCR analysis to establish standard curves and determine their regression equation. The average $\mathrm{C}_{\mathrm{T}}$ value for each target gene obtained from the same genotype were then used to calculate the absolute copy numbers for each $\mathrm{Gr}$ transcript in labella of the indicated genotypes. Absolute copy number of the target

gene $=($ copy number of the standard $) \times 10^{(\mathrm{Ct}-\mathrm{b}) / \mathrm{m}}$, where $\mathrm{b}$ and $\mathrm{m}$ refer to the slope and intercept of the standard curve regression equation, respectively. The number of transcripts in sweet GRNs was calculated by subtracting the number of transcripts in the Gr64f-GAL4 control (endogenous bitter Gr expression level) from the number of transcripts of flies ectopically expressing Grs. RNA extraction and quantitative PCR were performed at least three times.

Tip recording. 2-5-day-old flies housed in fresh vials were anesthetized by brief ice exposure. A glass capillary pipette containing Ringer's solution was inserted through the thorax to the base of the labellum and connected to ground to serve as the reference electrode. The indicated concentrations of each tastant compound were dissolved in $30 \mathrm{mM}$ tricholine citrate for electrical conductivity in a $10-20 \mu \mathrm{m}$ diameter recording electrode. After the recording electrode was connected to a TastePROBE (Syntech, Hilversum, The Netherlands), the electrical signals derived from taste sensilla were recorded using an acquisition controller (Syntech) attached to a computer. These signals were amplified $(\times 10)$, band-pass-filtered $(100-3000$ $\mathrm{Hz}$ ), and sampled at $12 \mathrm{kHZ}$. Neuronal firing rates were then analyzed using the Autospike 3.1 software (Syntech).

Two-way choice behavioral assay. Briefly, 40-50 3-5-day-old flies were collected under $\mathrm{CO}_{2}$ anesthesia at least 1 day prior to starvation. The flies were starved for $18 \mathrm{~h}$ in vials containing only $1 \%$ agarose gel. Then, they were given $90 \mathrm{~min}$ in a dark room at room temperature to choose between $1 \%$ agarose gel containing 1 $\mathrm{mM}$ sucrose or $5 \mathrm{mM}$ sucrose and the indicated concentrations of bitter chemicals. To monitor their food preference, each food was mixed with either a blue $(0.125$ $\mathrm{mg} / \mathrm{ml}$ Brilliant blue FCF) or red $(0.2 \mathrm{mg} / \mathrm{ml}$ sulforhodamine B) non-toxic dye. After freezing the flies, the color of their abdomens was scored under a stereomicroscope. A preference index was calculated using the following equation: Preference Index $(\mathrm{PI})=($ \# of red or blue abdomens $+1 / 2$ the \# of purple abdomens $) /$ Total \# of fed flies.

Whole-cell patch clamp recordings. Gr-and EGFP-expressing S2 cells on coverslips were transferred to a chamber positioned on the stage of an inverted microscope (IX73, Olympus, Tokyo, Japan). Whole-cell currents were measured using a multiclamp amplifier at a holding potential of $-60 \mathrm{mV}$ (Axon Instruments, Foster City, CA) at room temperature. The bath solution contained normal Ringer's solution: $140 \mathrm{mM} \mathrm{NaCl}, 5 \mathrm{mM} \mathrm{KCl}, 2 \mathrm{mM} \mathrm{CaCl}_{2}, 2 \mathrm{mM} \mathrm{MgCl}, 10 \mathrm{mM}$ HEPES (titrated to $\mathrm{pH} 7.4$ with $\mathrm{NaOH}$ ). The pipette solution contained $140 \mathrm{mM}$ CsCl, 5 mM EGTA, 10 mM HEPES, 2 mM MgATP, $0.2 \mathrm{mM} \mathrm{NaGTP} \mathrm{(titrated} \mathrm{to} \mathrm{pH}$ 7.2 with $\mathrm{CsOH}$ ). Electrodes were pulled from borosilicate glass to a final resistance of 2-4 M $\Omega$ after fire-polishing. The seal resistances were 3-10 G $\Omega$. After establishing a whole-cell configuration, currents were recorded in the presence of LOB, BER, DEN, CAF, STR, or SOA by applying $400 \mathrm{~ms}$ voltage-ramp pulses from -80 to $+80 \mathrm{mV}$ every $1500 \mathrm{~ms}$. Currents were digitized with a Digidata $1440 \mathrm{~A}$ converter (Axon Instruments), filtered at $5 \mathrm{kHz}$, and analyzed using Clampfit 10.2 (Axon Instruments)

Chemicals. Berberine, caffeine, denatonium, lobeline, sucrose, sucrose octacetate, strychnine, sulforhodamine B, tricholine citrate, and umbelliferone were purchased from Sigma-Aldrich (Saint Louis, MO). Brilliant blue FCF was purchased from Wako Pure Chemical Industries, Ltd (Osaka, Japan).

Statistics. All data were subjected to the Levene and Kolmogrov-Smirnov tests to evaluate variance homogeneity and normality, respectively. Depending on the results of these tests, ANOVAs with Tukey or Dunnet T3 post hoc tests or Kruskal -Wallis tests with Mann-Whitney $U$ post hoc tests were used to identify significant differences in comparisons between multiple groups. The unpaired Student's $t$-test or the Mann-Whitney $U$-test was used for comparisons between two groups.

Data availability. All relevant data are available from the authors upon request.
Received: 8 March 2017 Accepted: 4 October 2017

Published online: 14 November 2017

\section{References}

1. Masek, P. \& Scott, K. Limited taste discrimination in Drosophila. Proc. Nat Acad. Sci. USA 107, 14833-14838 (2010).

2. Behrens, M., Foerster, S., Staehler, F., Raguse, J. D. \& Meyerhof, W. Gustatory expression pattern of the human TAS2R bitter receptor gene family reveals a heterogenous population of bitter responsive taste receptor cells. J. Neurosci. 27, 12630-12640 (2007).

3. Weiss, L. A., Dahanukar, A., Kwon, J. Y., Banerjee, D. \& Carlson, J. R. The molecular and cellular basis of bitter taste in Drosophila. Neuron 69, 258-272 (2011).

4. Caicedo, A., Kim, K. N. \& Roper, S. D. Individual mouse taste cells respond to multiple chemical stimuli. J. Physiol. 544, 501-509 (2002).

5. Geran, L. C. \& Travers, S. P. Single neurons in the nucleus of the solitary tract respond selectively to bitter taste stimuli. J. Neurophysiol. 96, 2513-2527 (2006).

6. Stocker, R. F. The organization of the chemosensory system in Drosophila melanogaster: a review. Cell Tissue Res. 275, 3-26 (1994).

7. Vosshall, L. B. \& Stocker, R. F. Molecular architecture of smell and taste in Drosophila. Annu. Rev. Neurosci. 30, 505-533 (2007).

8. Hiroi, M., Marion-Poll, F. \& Tanimura, T. Differentiated response to sugars among labellar chemosensilla in Drosophila. Zoolog. Sci. 19, 1009-1018 (2002)

9. Kim, S. H. et al. Drosophila TRPA1 channel mediates chemical avoidance in gustatory receptor neurons. Proc. Natl Acad. Sci. USA 107, 8440-8445 (2010).

10. Kwon, Y. et al. Drosophila TRPA1 channel is required to avoid the naturally occurring insect repellent citronellal. Curr. Biol. 20, 1672-1678 (2010).

11. Zhang, Y. V., Raghuwanshi, R. P., Shen, W. L. \& Montell, C. Food experienceinduced taste desensitization modulated by the Drosophila TRPL channel. Nat. Neurosci. 16, 1468-1476 (2013).

12. Robertson, H. M., Warr, C. G. \& Carlson, J. R. Molecular evolution of the insect chemoreceptor gene superfamily in Drosophila melanogaster. Proc. Natl Acad. Sci. USA 100(Suppl 2): 14537-14542 (2003).

13. Scott, K. et al. A chemosensory gene family encoding candidate gustatory and olfactory receptors in Drosophila. Cell 104, 661-673 (2001).

14. Lee, Y. et al. Gustatory receptors required for avoiding the insecticide L-canavanine. J. Neurosci. 32, 1429-1435 (2012).

15. Lee, Y., Kim, S. H. \& Montell, C. Avoiding DEET through insect gustatory receptors. Neuron 67, 555-561 (2010).

16. Lee, Y., Moon, S. J. \& Montell, C. Multiple gustatory receptors required for the caffeine response in Drosophila. Proc. Natl Acad. Sci. USA 106, 4495-4500 (2009).

17. Lee, Y., Moon, S. J., Wang, Y. \& Montell, C. A Drosophila gustatory receptor required for strychnine sensation. Chem. Senses 40, 525-533 (2015).

18. Moon, S. J., Kottgen, M., Jiao, Y., Xu, H. \& Montell, C. A taste receptor required for the caffeine response in vivo. Curr. Biol. 16, 1812-1817 (2006).

19. Moon, S. J., Lee, Y., Jiao, Y. \& Montell, C. A Drosophila gustatory receptor essential for aversive taste and inhibiting male-to-male courtship. Curr. Biol. 19, 1623-1627 (2009).

20. Shim, J. et al. The full repertoire of Drosophila gustatory receptors for detecting an aversive compound. Nat. Commun. 6, 8867 (2015).

21. Delventhal, R. \& Carlson, J. R. Bitter taste receptors confer diverse functions to neurons. Elife 5 (2016).

22. Dahanukar, A., Lei, Y. T., Kwon, J. Y. \& Carlson, J. R. Two Gr genes underlie sugar reception in Drosophila. Neuron 56, 503-516 (2007).

23. Jiao, Y., Moon, S. J. \& Montell, C. A Drosophila gustatory receptor required for the responses to sucrose, glucose, and maltose identified by mRNA tagging. Proc. Natl Acad. Sci. USA 104, 14110-14115 (2007).

24. Jiao, Y., Moon, S. J., Wang, X., Ren, Q. \& Montell, C. Gr64f is required in combination with other gustatory receptors for sugar detection in Drosophila. Curr. Biol. 18, 1797-1801 (2008).

25. Slone, J., Daniels, J. \& Amrein, H. Sugar receptors in Drosophila. Curr. Biol. 17, 1809-1816 (2007).

26. Gong, W. J. \& Golic, K. G. Ends-out, or replacement, gene targeting in Drosophila. Proc. Natl Acad. Sci. USA 100, 2556-2561 (2003).

27. Xiang, Y. et al. Light-avoidance-mediating photoreceptors tile the Drosophila larval body wall. Nature 468, 921-926 (2010).

28. Ling, F., Dahanukar, A., Weiss, L. A., Kwon, J. Y. \& Carlson, J. R. The molecular and cellular basis of taste coding in the legs of Drosophila. J. Neurosci. 34, 7148-7164 (2014).

29. Jeong, Y. T. et al. Mechanosensory neurons control sweet sensing in Drosophila. Nat. Commun. 7, 12872 (2016) 


\section{Acknowledgements}

We thank the Bloomington Stock Center and Drs H. Amrein and J. Carlson for fly stocks. We thank Dr J. Shim, Mr. J. Choi, Ms. H. S. Choi, and Miss H. Na for helping generate mutants and transgenic flies. This work was supported by a National Research Foundation of Korea (NRF) Grant funded by the Korean Government (MSIP) (NRF2016R1A5A2008630 and NRF-2012M3A9B2052524 to S.J.M., NRF-

2016R1D1A1B03932743 to J.Y.K.)

\section{Author contributions}

H.Y.S. performed the in vivo electrophysiological experiments and analyzed the resulting data. Y.T.J. performed the behavioral assays and analyzed the resulting data. H.K. and S.M.O helped with the experiments for the revised version of this manuscript. J.Y.L. and S.W.H. performed the patch clamp experiments and analyzed the resulting data. J.Y.K. and S.J.M. supervised the project and wrote the paper.

\section{Additional information}

Supplementary Information accompanies this paper at doi:10.1038/s41467-017-01639-5.

Competing interests: The authors declare no competing financial interests.

Reprints and permission information is available online at http://npg.nature.com/ reprintsandpermissions/

Publisher's note: Springer Nature remains neutral with regard to jurisdictional claims in published maps and institutional affiliations.

Open Access This article is licensed under a Creative Commons Attribution 4.0 International License, which permits use, sharing, adaptation, distribution and reproduction in any medium or format, as long as you give appropriate credit to the original author(s) and the source, provide a link to the Creative Commons license, and indicate if changes were made. The images or other third party material in this article are included in the article's Creative Commons license, unless indicated otherwise in a credit line to the material. If material is not included in the article's Creative Commons license and your intended use is not permitted by statutory regulation or exceeds the permitted use, you will need to obtain permission directly from the copyright holder. To view a copy of this license, visit http://creativecommons.org/ licenses/by/4.0/.

(c) The Author(s) 2017 